\title{
Even Moments and Moment-Based Distribution Functions of the Porod-Kratky Chain
}

\author{
Kazuo NAGAI \\ Government Industrial Research Institute, Osaka, \\ Midorigaoka 1, Ikeda, Osaka, Japan.
}

(Received March, 17, 1972)

\begin{abstract}
A method is developed for calculating the even moments $\left\langle r^{2 k}\right\rangle$ of the end-to-end distance $r$ of the Porod-Kratky wormlike chain, by adapting Hermans and Ullman's recurrence-formula method to computer calculations. Actual calculations are extended to $\left\langle r^{40}\right\rangle$. The whole range of the argument $t=L /(2 a)$, ( $L$ and $a$ being the contour and persistent lengths, respectively), is covered with an accuracy of $10^{-5}$ for up to $\left\langle r^{12}\right\rangle$. For higher moments there remains a range of $t$ which is not effectively covered because of a loss of accuracy due to a serious cancellation in computational results. A good agreement is achieved by a proper adjustment of parameters between even moments (through $\left\langle\boldsymbol{r}^{12}\right\rangle$ ) of the Porod-Kratky chain and of a realistic model for polyethylene. Exact distribution functions and some averages are calculated for $t>10$ with recourse to a previously developed method of the moment-based distribution function. Daniels' distribution is found to be a satisfactory approximation. A further improved, closed distribution function is derived.

KEY WORDS Porod-Kratky Wormlike Chain / End-to-End Distance / Even Moments / Moment-Based Distribution Function / Daniels' Distribution /
\end{abstract}

For semifiexible polymer chains such as polypeptides and polynucleotides in helicoidal conformations in solution, the Porod-Kratky wormlike chain (the PK chain) has long been considered to be a good model for describing their average spatial dimensions, specifically the mean-square end-to-end distance $\left\langle r^{2}\right\rangle$ and the mean-square radius of gyration. Much work has therefore been published on the theoretical study on these and other more complex quantities of the chain and on the application thereof to the analysis of experimental data. Recently it was demonstrated by Maeda, Saito, and Stockmayer ${ }^{2}$ that the PK chain is a good model for typical flexible polymer chains of short length as well, if a proper adjustment of parameters is made (see also seq.).

The PK chain is not a tractable one. Its second moment was calculated by Porod and Kratky themselves, ${ }^{1}$ and the fourth and sixth moments by Hermans and Ullman ${ }^{3}$ and Heine, Kratky, Porod, and Schmitz. ${ }^{4}$ Higher even moments have not yet been calculated, in contrast with realistic chain models for which even moments up to $\left\langle r^{12}\right\rangle$ were computed. ${ }^{5} \quad$ No closed, exact expression for the distribution function has been reported, and there is little chance of finding one. An approximate expression only was reported by Daniels. ${ }^{6}$

In an elaboration of the PK chain the author attempted to calculate even moments as high as possible, and has actually reached as far as $\left\langle r^{40}\right\rangle$. The underlying method is to solve with a computer a recurrence formula for Laplace transforms of even and other related moments, which was previously derived and solved manually by Hermans and Ullman. ${ }^{3}$ Even moments thus obtained are compared with those for realistic chain models, thereby gauging the usefulness of the PK chain. Moment-based distribution functions and some averages are calculated from these even moments by a method previously developed, ${ }^{5,7,8}$ and are compared with those of Daniels. ${ }^{6}$ An improved distribution function is derived by using the newly calculated eighth moment. 


\section{THEORY}

\section{Even Moments; Computer Adaptation of Hermans} and Ullman's Method

Hermans and Ullman ${ }^{3}$ derived a differential equation for the distribution function for the displacement $\mathbf{r}$ of one end and the tangent $\boldsymbol{\omega}=$ $(\alpha, \beta, \gamma)$ at that end with respect to the other end, for the PK chain of contour length $t$. This differential equation was, ${ }^{3}$ and has yet been, unsoluble analytically, but they derived therefrom the following recurrence formula:

$$
a_{m} u_{m}^{(k)}=2(k-m) u_{m+1}^{(k)}+m u_{m-1}^{(k-1)}+m(m-1) u_{m-2}^{(k-1)}
$$

or

$$
\begin{aligned}
u_{m}{ }^{(k)}= & a_{m}{ }^{-1}\left[2(k-m) u_{m+1}^{(k)}+m u_{m-1}^{(k-1)}\right. \\
& \left.+m(m-1) u_{m-2}^{(k-1)}\right]
\end{aligned}
$$

where

$$
a_{m}=p+m(m+1)
$$

and $u_{m}{ }^{(k)}$ is the dimensionless Laplace transform with respect to $t$ of the average $\left\langle(\boldsymbol{\omega} \cdot \mathbf{r})^{m} \boldsymbol{r}^{2(k-m)}\right\rangle$, (here $r \equiv|\mathbf{r}|$ ):

$$
\begin{aligned}
u_{m}{ }^{(k)} & =\mathrm{L}_{\mathrm{d} 1}\left[\left\langle(\boldsymbol{\omega} \cdot \mathbf{r})^{m} r^{2(k-m)}\right\rangle\right] \\
& =p \int_{0}^{\infty}\left\langle(\boldsymbol{\omega} \cdot \mathbf{r})^{m} r^{2(k-m)}\right\rangle \mathrm{e}^{-p t} \mathrm{~d} t
\end{aligned}
$$

The dimensionless Laplace transform is related to the ordinary Laplace transform by

$$
F(p)=\mathrm{L}_{\mathrm{d} 1}[f(t)]=p \mathrm{~L}_{\mathrm{od}}[f(t)]
$$

as is apparent from eq 4 , and hence

$$
f(t)=\mathrm{L}_{\text {od }}^{-1}\left[p^{-1} F(p)\right]
$$

Therefore one can calculate $\left\langle r^{2 k}\right\rangle$ through

$$
\left\langle r^{2 k}\right\rangle=\mathrm{L}_{\text {od }}^{-1}\left[p^{-1} u_{0}{ }^{(k)}\right]
$$

It must noted that in the derivation of eq 1 Hermans end Ullman ${ }^{3}$ used the reduced variables, i.e., $\mathbf{r}$ and $t$ both measured in units of $2 a$ where $a$ is the persistent length.

Hermans and Ullman ${ }^{3}$ solved eq 1 for $u_{0}^{(1)}$, $u_{0}{ }^{(2)}$, and $u_{0}{ }^{(3)}$, and inverted the first two to obtain $\left\langle r^{2}\right\rangle$ and $\left\langle r^{4}\right\rangle$. Heine, et al., ${ }^{4}$ inverted $u_{0}{ }^{(3)}$ to obtain $\left\langle r^{6}\right\rangle$. These calculations were all made manually. This approach becomes prohibitively difficult for higher moments because of the tremendous increase in the number of calculations involved. The author therefore attempted to solve eq 1 with a computer. This method also meets with a difficulty because eq 1 involves the Laplace-transform parameter $p$ while a computer treats only numerical values. The difficulty can be surpassed by the following device. Even moments are expressed in two forms which are alternative in a theoretical sense but supplementary practically.

(i) Exponential Series Expression. This expression is a familiar one, being a finite series whose general term is expressed $t^{j-1} \exp [-l(l+$ 1) $t$ ]. This ${ }^{*}$ expression is effective for large $t$.

For the adaptation of eq 1 to the computer calculation the explicit display of first severals of $u_{m}{ }^{(k)}$ is instructive:

$$
\begin{gathered}
u_{0}{ }^{(0)}=1 \\
u_{1}^{(1)}=a_{1}^{-1} \text { and } u_{0}^{(1)}=a_{0}^{-1}-a_{1}^{-1} \\
p^{-1} u_{0}^{(1)}=a_{0}^{-2}-\frac{1}{2} a_{0}^{-1}+\frac{1}{2} a_{1}^{-1}
\end{gathered}
$$

$$
\left.\begin{array}{c}
u_{2}^{(2)}=\frac{1}{3} a_{0}^{-1}-\frac{1}{3} a_{2}^{-1} \\
u_{1}^{(2)}=\frac{5}{6} a_{0}^{-1}-a_{1}^{-2}-a_{1}^{-1}+\frac{1}{6} a_{2}^{-1} \\
u_{0}^{(2)}=\frac{10}{3} a_{0}^{-2}-\frac{26}{9} a_{0}^{-1}+2 a_{1}^{-2}+3 a_{1}^{-1}-\frac{1}{9} a_{2}^{-1}
\end{array}\right\}
$$

$$
\begin{aligned}
p^{-1} u_{0}{ }^{(3)}= & \frac{70}{3} a_{0}^{-4}-\frac{259}{9} a_{0}^{-3}+\frac{226}{9} a_{0}^{-2}-\frac{6143}{324} a_{0}^{-1} \\
& +\frac{21}{5} a_{1}^{-3}+\frac{639}{50} a_{1}^{-2}+\frac{4743}{250} a_{1}^{-1} \\
& -\frac{1}{54} a_{2}^{-2}-\frac{1}{81} a_{2}^{-1}+\frac{1}{4500} a_{3}^{-1}
\end{aligned}
$$

Equations $8-13$ suggest that $u_{m}{ }^{(k)}$ and $p^{-1} u_{0}{ }^{(k)}$ are expressed

$$
\begin{gathered}
u_{m}{ }^{(k)}=\sum_{l=0}^{k} \sum_{j=k-l+1}^{1} c_{k m l j}^{\prime \prime} a_{l}^{-j} \\
p^{-1} u_{0}^{(k)}=\sum_{l=0}^{k} \sum_{j=k-l+1}^{1} c_{k l j}^{\prime} a_{l}^{-j}
\end{gathered}
$$

where $c_{k m l j}^{\prime \prime}$ and $c_{k l j}^{\prime}$ are numerical constants dependent on their arguments. $u_{m}{ }^{(k)}$ is expressed in a computer as an array of $\frac{1}{2}(k+1)(k+2)$ computer words for $c_{k m l j}^{\prime \prime}$ arranged in the order as 
implied in eq 14, and similarly with $p^{-1} u_{0}{ }^{(k)}$. Assuming one has already constructed such arrays for $u_{m+1}^{(k)}, \boldsymbol{u}_{m-1}^{(k-1)}$, and $u_{m-2}^{(k-1)}$, two arrays each of $\frac{1}{2}(k+1)(k+2)$ words are reserved in the computer; one is for $u_{m}^{(k)}$ and the other is a working array. First one constructs $a_{m} u_{m}{ }^{(k)}$ on this working array according to eq 1 . The calculation involved is simply to add the three $c^{\prime \prime}$ s $s$ corresponding to the same $a_{l}^{-j}$ term, with appropriate factors (as given in eq 1) multiplied. Namely, if one writes

$$
a_{m} u_{m}{ }^{(k)}=\sum_{l=0}^{k} \sum_{j=k-l+1}^{1} c_{k m l j}^{(3)} a_{l}^{-j}
$$

then we have

$$
\begin{aligned}
c_{k m l j}^{(3)}= & 2(k-m) c_{k, m+1, l j}^{\prime \prime}+m c_{k-1, m-1, l j}^{\prime \prime} \\
& +m(m-1) c_{k-1, m-2, l j}^{\prime \prime}
\end{aligned}
$$

The next step is to construct $u_{m}{ }^{(k)}$ from this $a_{m} u_{m}{ }^{(k)}$ according to eq 2 . After division of eq 16 by $a_{m}$, the terms in its rhs, having the general form $c_{k m l j}^{(3)} a_{m}{ }^{-1} a_{l}{ }^{-j}$, are categorized into two groups: one for $m=l$ and the other for $m \neq l$. Consider initially the first group of terms. Since the latter group of terms do not yield terms of the form $a_{l}^{-j}(j>1)$ upon decomposition into partial fractions (see below), one finds

$$
c_{k m m j}^{\prime \prime}=c_{k m m, j-1}^{(3)}, \quad j=k-m+1, \ldots, 3,2
$$

Consider now the second group terms. Using the identity

$$
\begin{aligned}
a_{m}^{-1} a_{l}^{-j}= & -\left[\left(a_{l}-a_{m}\right)^{-1} a_{l}^{-j}\right. \\
& +\left(a_{l}-a_{m}\right)^{-2} a_{l}^{-(j-1)}+\cdots \\
& \left.+\left(a_{l}-a_{m}\right)^{-j} a_{l}^{-1}\right]+\left(a_{l}-a_{m}\right)^{-j} a_{m}{ }^{-1}
\end{aligned}
$$

One rearranges

$$
\sum_{j=k-l+1}^{1} c_{k m l j}^{(3)} a_{m}{ }^{-1} a_{l}^{-j}=\sum_{j=k-l+1}^{1} c_{k m l j}^{\prime \prime} a_{l}^{-j}-c_{k m l 1}^{\prime \prime} a_{m}{ }^{-1}
$$

where

$$
\begin{aligned}
\boldsymbol{c}_{k m l j}^{\prime \prime}= & -\left[\left(\boldsymbol{a}_{l}-\boldsymbol{a}_{m}\right)^{-1} \boldsymbol{c}_{k m l j}^{(3)}+\left(\boldsymbol{a}_{l}-\boldsymbol{a}_{m}\right)^{-2} \boldsymbol{c}_{k m l, j+1}^{(3)}+\cdots\right. \\
& \left.+\left(\boldsymbol{a}_{l}-\boldsymbol{a}_{m}\right)^{-(k-l-j+2)} \boldsymbol{c}_{k m l, k-l+1}^{(3)}\right]
\end{aligned}
$$

or

$$
\begin{aligned}
c_{k m l j}^{\prime \prime}=\left(a_{l}-a_{m}\right)^{-1}\left(c_{k m l, j+1}^{\prime \prime}-c_{k m l j}^{(3)}\right), & \\
& j=k-l+1, \ldots, 2,1
\end{aligned}
$$

with $c_{k m l, k-l+2}^{\prime \prime} \equiv 0$. According to eq 20, $c_{k m m l}^{\prime \prime}$, which is not comprehended in eq 18 , is given by

$$
c_{k m m 1}^{\prime \prime}=-\sum_{l \neq m} c_{k m l 1}^{\prime \prime}
$$

Thus the rule to construct $u_{m}{ }^{(k)}$ from $a_{m} u_{m}{ }^{(k)}$ is established; eq 18, 21, and 22. The passage from $u_{0}{ }^{(k)}$ to $p^{-1} u_{0}{ }^{(k)}$ is similar to the special case of the above described one, i.e., from $u_{1}{ }^{(k)}$ to $u_{0}{ }^{(k)}$.

The set of $u_{m}{ }^{(k)}$ 's for $m=0,1, \ldots, k$ requires too much information to be stored in the core memory simultaneously. Two magnetic tapes are used. Let the arrays for $u_{k-1}^{(k-1)}, u_{k-2}^{(k-1)}, \ldots, u_{0}^{(k-1)}$ have already been computed and stored in this order in one of two tapes. Then, $u_{k-1}^{(k-1)}$ and $u_{k-2}^{(k-1)}$ are read into the computer, and $u_{k}^{(k)}$ is calculated from them and written out in the other tape. $\boldsymbol{u}_{k-3}^{(k-1)}$ is read in, the now unnecessary $\boldsymbol{u}_{k-1}^{(k-1)}$ being erased, and $u_{k-1}^{(k)}$ is calculated and written out. This procedure is repeated until $u_{0}{ }^{(k)}$ is calculated and written out. At this point $p^{-1} u_{0}{ }^{(k)}$ is calculated and stored in either tape. $u_{m}^{(k+1)}$ 's are then calculated by using $u_{m}{ }^{(k)}$ 's just constructed. In this procedure the roles of two tapes are exchanged and $u_{m}{ }^{(k+1)}$,s are stored in the first tape the unnecessary $u_{m}{ }^{(k-1)}$,s therefore being erased. Ultimately one has in one of tapes the arrays for $p^{-1} u_{0}^{(0)}, p^{-1} u_{0}{ }^{(1)}, \ldots, p^{-1} u_{0}^{(k)}$, from which one can readily calculate even moments through $\left\langle r^{2 k}\right\rangle$. The starting $u$ is $u_{1}{ }^{(1)}$, the array for it being expressed according to eq 9

$$
u_{1}{ }^{11)}:\left(\begin{array}{lll}
0 & 0 & 1
\end{array}\right)
$$

$c_{k m l j}^{(3)}, c_{k m l j}^{\prime \prime}, c_{k l j}^{\prime}$ (and $c_{k l j}$ below) are found to vary between very small and large values for large $k$ (see Table I for $c_{k l j}$ ). An appropriate factor multiplied to all these $c$ 's is necessary to prevent overflow and underflow. The factor $10^{-k+2 l}$ is found to be appropriate, the $10^{-k+2 l} c$ remaining within $10^{ \pm 50}$ up to $k=20$.

Even moments are calculated through

$$
\left\langle r^{2 k}\right\rangle=\mathrm{L}_{\text {od }}^{-1}\left[p^{-1} u_{0}^{(k)}\right]=\sum_{l=0}^{k} \sum_{j=k-l+1}^{1} c_{k l j} t^{j-1} \mathrm{e}^{-l(l+1) t}
$$

with

$$
c_{k l j}=c_{k l j}^{\prime} /(j-1) !
$$

where the following theorem is used:

$$
\begin{aligned}
\mathrm{L}_{\text {od }}^{-1}\left[(p+b)^{-j}\right]= & \frac{1}{(j-1) !} t^{j-1} \mathrm{e}^{-b t} \\
& \text { with } j \geq 1 \text { and } b \geq 0
\end{aligned}
$$

From eq 10, 12, and 13 one immediately obtains 
Table I. $c_{k l j}$ in eq 24 for $\left\langle r^{8}\right\rangle,\left\langle r^{10}\right\rangle$, and $\left\langle r^{12}\right\rangle^{\mathrm{a}}$

\begin{tabular}{|c|c|c|c|c|c|c|c|}
\hline$\lambda$ & 7 & 6 & 5 & 4 & 3 & 2 & 1 \\
\hline & & & \multicolumn{5}{|c|}{$c_{4 l j}$} \\
\hline 0 & & & 21166666666 & -27466666666 & 32369333333 & -34165674074 & 33290746666 \\
\hline 1 . & & & & -15039999999 & -26177599999 & -32416671999 & -33290554844 \\
\hline 2 & & & & & $\overline{1} 1587301587$ & $\overline{2} 8314436884$ & $-\overline{1} 1911240687$ \\
\hline 3 & & & & & & $-\overline{3} 1777777777$ & $-\overline{4} 7111111111$ \\
\hline 4 & & & & & & & $\overline{5} 1295756397$ \\
\hline & & \multicolumn{6}{|c|}{$c_{5 l j}$} \\
\hline 0 & & 24277777777 & -34206481481 & 42090135802 & -46285698765 & 51109826353 & -48999555774 \\
\hline 1 & & & 21386000000 & 32843279999 & 42087929485 & 46901139925 & 48999453333 \\
\hline 2 & & & & $-\overline{1} 1385739480$ & $\overline{2} 593888349$ & 01177750011 & 0102441966 \\
\hline 3 & & & & & $\overline{3} 1086419753$ & $\overline{4} 66941015$ & $-\overline{6} 75598$ \\
\hline 4 & & & & & & $-\overline{6} 925540283$ & $-\overline{6} 2644401$ \\
\hline 5 & & & & & & & $\overline{8} 4443609$ \\
\hline \multicolumn{8}{|c|}{$c_{6 l j}$} \\
\hline 0 & 31853703703 & -42595185185 & 51850790740 & -58331792016 & 62437762355 & -64315409898 & 63559443112 \\
\hline 1 & & -24324319999 & -41334361599 & -51574336845 & -59258383197 & -62803474356 & -63559442505 \\
\hline 2 & & & 11286758089 & $-\overline{1} 2818612957$ & -0319190808 & -043991907 & $-\overline{1} 6063456$ \\
\hline 3 & & & & $-\overline{4} 6277091906$ & $-\overline{4} 4070599$ & $-\overline{4} 138378$ & $-\overline{4} 38993$ \\
\hline 4 & & & & & $\overline{6} 468780143$ & $\overline{6} 2273185$ & $\overline{7} 1443$ \\
\hline 5 & & & & & & $-\overline{8} 2962406$ & $-\overline{9} 6583$ \\
\hline 6 & & & & & & & $\overline{10} 100156$ \\
\hline
\end{tabular}

a Values are expressed in floating form: a bcde $\equiv 0 . b c d e \times 10^{\mathrm{a}} ;-\overline{\mathrm{a}} \mathrm{bcde} \equiv-0 . \mathrm{bcde} \times 10^{-\mathrm{a}}$.

the well-known ${ }^{1,3,4}$ results

$$
\begin{gathered}
\left\langle r^{2}\right\rangle=t-\frac{1}{2}+\frac{1}{2} \mathrm{e}^{-2 t} \\
\left\langle r^{4}\right\rangle=\frac{5}{3} t^{2}-\frac{26}{9} t+\frac{107}{54}-t \mathrm{e}^{-2 t}-2 \mathrm{e}^{-2 t}+\frac{1}{54} \mathrm{e}^{-6 t} \\
\left\langle r^{6}\right\rangle=\frac{35}{9} t^{3}-\frac{259}{18} t^{2}+\frac{226}{9} t-\frac{6143}{324} \\
+\frac{21}{10} t^{2} \mathrm{e}^{-2 t}+\frac{639}{50} t \mathrm{e}^{-2 t}+\frac{4743}{250} \mathrm{e}^{-2 t} \\
-\frac{1}{54} t \mathrm{e}^{-6 t}-\frac{1}{81} \mathrm{e}^{-6 t}+\frac{1}{4500} \mathrm{e}^{-12 t}
\end{gathered}
$$

These relations are used to check the validity of the programing.

(ii) Power Series Expression. $u_{m}{ }^{(k)}$ and $p^{-1} u_{0}{ }^{(k)}$ are expressed in power series in $p^{-1}$ and hence $\left\langle r^{2 k}\right\rangle$ is in a power series in $t$. This expression is effective and limited for small $t$.

To find an appropriate way of adaptation to a computer it is advisable to write explicitly first severals of $u_{m}{ }^{(k)}$, Expanding eq 9-10 one has

$$
\left.\begin{array}{c}
u_{1}^{(1)}=p^{-1} \sum_{n=0}^{\infty}(-1)^{n} 2^{n} p^{-n} \\
u_{0}^{(1)}=2 p^{-2} \sum_{n=0}^{\infty}(-1)^{n} 2^{n} p^{-n} \\
u_{2}^{(2)}=2 p^{-2} \sum_{n=0}^{\infty}(-1)^{n} 6^{n} p^{-n} \\
u_{1}^{(2)}=3 ! p^{-3} \sum_{n=0}^{\infty}(-1)^{n} 6^{n}\left[1+n 3^{-(n+1)}\right] p^{-n} \\
u_{0}^{(2)}=4 ! p^{-4} \sum_{n=0}^{\infty}(-1)^{n} 6^{n}\left[1+n 3^{-(n+1)}\right] p^{-n}
\end{array}\right\}
$$

These expressions suggest that $u_{m}{ }^{(k)}$ is of the form $u_{m}^{(k)}=(2 k-m) ! p^{-(2 k-m)} \sum_{n=0}^{\infty}(-1)^{n}[k(k+1)]^{n} f_{k m n} p^{-n}$

Substitution of eq 32 into eq 1 yields the following recurrence relation for $f_{k m n}$ :

$$
\begin{aligned}
f_{k m n}= & m(m+1)[k(k+1)]^{-1} f_{k m, n-1} \\
& +2(k-m)(2 k-m)^{-1} f_{k, m+1, n} \\
& +m(2 k-m)^{-1}[(k-1) /(k+1)]^{n} f_{k-1, m-1, n} \\
& -m(m-1)[(k-1) /(k+1)]^{n} \\
& \times[k(k-1)]^{-1} f_{k-1, m-2, n-1}
\end{aligned}
$$


The factor $(2 k-m)$ ! in eq 32 makes $f_{k m 0}=1$. This is readily seen as follows. From eq 33

$$
\begin{aligned}
f_{k m 0}= & 2(k-m)(2 k-m)^{-1} f_{k, m+1,0} \\
& +m(2 k-m)^{-1} f_{k-1, m-1,0}, \\
f_{k k 0}= & \text { for } m \neq k
\end{aligned}
$$

From eq 34 one finds that if $f_{k-1, m_{0}}=1$ then $f_{k m 0}=1$. $f_{100}=f_{110}=1$ according to eq 30 . This completes the mathematical-induction proof for $f_{k m 0}=1$ for all $k$ and $m$. Calculation with a computer indicates that $f_{k m n}$, starting with unity at $n=0$, either remains unchanged, decreases, or first increases and then decreases, with increasing $n$; ultimately it approaches an asymptotic positive value smaller than unity. All $f_{k m n}$ are positive and do not exceed 1.5 for up to $k=30$.

The method of computer calculations is similar to case (i) above, with the only difference that the array for $u_{m}{ }^{(k)}$ includes $f_{k m n}\left(n=0,1, \cdots, n_{\mathrm{M}}\right)$. It is impossible to store an infinite number of $f_{k m n}$, so one is obliged to truncate the series at some $n, n_{\mathrm{M}}$. In the present calculation $n_{\mathrm{M}}=499$ was used for all $k$ and $m$, this choice being made in consideration of the size of core memories of the computer used. This truncation of the series does not introduce errors in $f_{k m n}$ because the subscripts to $f$ in the rhs of eq 33 do not include $n+1, n+2, \ldots$. The rule to construct $f_{k m n}$, i.e., through eq 33, is much simpler than that for $\boldsymbol{c}_{k m l j}^{\prime \prime}$ in case (i). The starting $u$ is $u_{0}{ }^{(0)}$, the array for it being, according to eq 8 and 32,

$$
u_{0}^{(0)}:\left(\begin{array}{lllll}
1 & 0 & 0 & \cdots & 0
\end{array}\right)
$$

Even moments are expressed

$\left\langle r^{2 k}\right\rangle=(2 k) ! t^{2 k} \sum_{n=0}^{\infty}(-1)^{n}[(2 k+n) !]^{-1}[k(k+1)]^{n} f_{k 0 n} t^{n}$

$\left\langle r^{4}\right\rangle$ and $\left\langle r^{6}\right\rangle$ in this form were obtained by Heine, et al., ${ }^{4}$ by expanding eq 28 and 29 . (In eq 5 and 6 of ref 4 the summation should start with $n=0$ in place of $n=4$ and 6.)

Expressions in terms of more familiar nonreduced parameters, persistent length $a$ and contour length $L$, are obtained by replacing $\left\langle r^{2 k}\right\rangle$ with $\left\langle r^{2 k}\right\rangle /(2 a)^{2 k}$ and $t$ with $L /(2 a)$.

\section{Moments-Based Distribution Functions and Some Averages}

The distribution function $W_{t *}(\mathbf{r})$ can be ex- pressed exactly in terms of even moments: ${ }^{5,7}$

$$
\begin{aligned}
W_{t *}(\mathbf{r})= & \left(\frac{2}{3} \pi\left\langle r^{2}\right\rangle_{*}\right)^{-3 / 2} \exp \left(-\frac{3}{2} r^{2} /\left\langle r^{2}\right\rangle_{*}\right) \\
& \times\left\{1+9\left\langle r^{2}\right\rangle_{*}{ }^{-2}\left(r^{2}-\left\langle r^{2}\right\rangle_{*}\right) g_{2}{ }^{\prime}\right. \\
& +9^{2}\left\langle r^{2}\right\rangle_{*}{ }^{-4}\left(r^{4}-\frac{10}{3} r^{2}\left\langle r^{2}\right\rangle_{*}+\frac{5}{3}\left\langle r^{2}\right\rangle_{*}{ }^{2}\right) g_{4}{ }^{\prime} \\
& +\cdots+9^{k}\left\langle r^{2}\right\rangle_{*}{ }^{-2 k} \sum_{m=0}^{k}(-1)^{m}(2 k+1) ! \\
& \times\left[(2 k-2 m+1) ! m ! 6^{m}\right]^{-1} r^{2(k-m)} \\
& \left.\times\left\langle r^{2}\right\rangle_{*}{ }^{m} g_{2 k}^{\prime}+\cdots\right\}
\end{aligned}
$$

where $\left\langle r^{2}\right\rangle_{*}$ is such part of $\left\langle r^{2}\right\rangle$ as proportional to the chain length, being for the PK chain

$$
\left\langle r^{2}\right\rangle_{*}=t \lim _{t \rightarrow \infty} t^{-1}\left\langle r^{2}\right\rangle=t
$$

and

$$
\begin{aligned}
g_{2}{ }^{\prime}= & (1 / 3 !)\left(\left\langle r^{2}\right\rangle-\left\langle r^{2}\right\rangle_{*}\right) \\
g_{4}{ }^{\prime}= & (1 / 5 !)\left(\left\langle r^{4}\right\rangle-\frac{10}{3}\left\langle r^{2}\right\rangle\left\langle r^{2}\right\rangle_{*}+\frac{5}{3}\left\langle r^{2}\right\rangle_{*}{ }^{2}\right), \ldots, \\
g_{2 k}^{\prime}= & \sum_{m=0}^{k}(-1)^{m}\left[(2 k-2 m+1) ! m ! 6^{m}\right]^{-1} \\
& \times\left\langle r^{2(k-m)}\right\rangle\left\langle r^{2}\right\rangle_{*}^{m}, \ldots
\end{aligned}
$$

The value of $W_{t *}(\mathbf{r})$ at $\mathbf{r}=0, W_{t *}(0)$, and the average of $r^{-1},\left\langle r^{-1}\right\rangle_{*}$, are of particular physical interest. There is no direct analytical method to calculate them in contrast with even moments, but they can be expressed in terms of even moments:

$$
\begin{aligned}
W_{t *}(0)= & \left(\frac{2}{3} \pi\left\langle r^{2}\right\rangle_{*}\right)^{-3 / 2}\left[1-9\left\langle r^{2}\right\rangle_{*}{ }^{-1} g_{2}{ }^{\prime}\right. \\
& +135\left\langle r^{2}\right\rangle_{*}{ }^{-2} g_{4}{ }^{\prime}-\cdots+(-1)^{k}(2 k+1) ! \\
& \left.\times(k !)^{-1}(3 / 2)^{k}\left\langle r^{2}\right\rangle_{*}{ }^{-k} g_{2 k}^{\prime}+\cdots\right] \\
\left\langle r^{-1}\right\rangle_{*}= & \left(\frac{1}{6} \pi\left\langle r^{2}\right\rangle_{*}\right)^{-1 / 2}\left[1-3\left\langle r^{2}\right\rangle_{*}{ }^{-1} g_{2}{ }^{\prime}\right. \\
& +27\left\langle r^{2}\right\rangle_{*}{ }^{-2} g_{4}{ }^{\prime}-\cdots+(-1)^{k}(2 k) ! \\
& \left.(k !)^{-1}(3 / 2)^{k}\left\langle r^{2}\right\rangle_{*}{ }^{-k} g_{2 k}^{\prime}+\cdots\right]
\end{aligned}
$$

For graphical comparisons one uses, in place of $W_{t *}(\mathbf{r})$, the reduced distribution function

$$
\boldsymbol{P}_{t *}(\xi)=4 \pi\left\langle r^{2}\right\rangle{ }^{3 / 2} \xi^{2} W_{t *}\left(\left\langle r^{2}\right\rangle{ }^{1 / 2} \xi\right)
$$

where $\xi=\left\langle r^{2}\right\rangle_{*}{ }^{-1 / 2} \mathbf{r}$ and $\xi=|\boldsymbol{\xi}|$. Hence 


$$
\begin{aligned}
P_{t *}(\xi)= & 4 \pi\left(\frac{2}{3} \pi\right)^{-3 / 2} \xi^{2} \exp \left(-\frac{3}{2} \xi^{2}\right) \\
& \times\left\{1+9\left\langle r^{2}\right\rangle_{*}{ }^{-1} g_{2}{ }^{\prime}\left(\xi^{2}-1\right)+9^{2}\left\langle r^{2}\right\rangle_{*}{ }^{-2} g_{4}{ }^{\prime}\right. \\
& \times\left(\xi^{4}-\frac{10}{3} \xi^{2}+\frac{5}{3}\right)+\cdots+9^{k}\left\langle r^{2}\right\rangle_{*}{ }^{-k} g_{2 k}^{\prime} \\
& \times \sum_{m=0}^{k}(-1)^{m}(2 k+1) ![(2 k-2 m+1) ! \\
& \left.\left.\times m ! 6^{m}\right]^{-1} \xi^{2(k-m)}+\cdots\right\}
\end{aligned}
$$

In actual calculations one is obliged to truncate the series insofar as it is not possible to calculate all even moments. $W_{t *}(\mathbf{r}), W_{t *}(0),\left\langle r^{-1}\right\rangle_{*}$, and $\boldsymbol{P}_{t *}(\xi)$, truncated at the $k$-th term, i.e., involving $g_{2 k}^{\prime}$, are written as $W_{t *}^{(k)}(\mathbf{r}), W_{t *}^{(k)}(0),\left\langle r^{-1}\right\rangle_{*}^{(k)}$, and $\boldsymbol{P}_{t *}^{(k)}(\xi)$, respectively.

Jernigan and Flory ${ }^{9,10}$ proposed a modified distribution function and suggested that it was preferable for reasons of rapid convergence and others. It is

$W_{t}(\mathbf{r})=W_{t *}(\mathbf{r})$ in eq 37 , with $\left\langle r^{2}\right\rangle$ in place of $\left\langle r^{2}\right\rangle_{*}$

Correspondingly one has

$W_{t}(0)=W_{t *}(0)$ in eq 40 , with $\left\langle r^{2}\right\rangle$ in place of $\left\langle r^{2}\right\rangle_{*}$

$\left\langle r^{-1}\right\rangle=\left\langle r^{-1}\right\rangle_{*}$ in eq 41 , with $\left\langle r^{2}\right\rangle$ in place of $\left\langle r^{2}\right\rangle_{*}$

$P_{t}(\xi)=P_{t *}(\xi)$ in eq 43 , with $\left\langle r^{2}\right\rangle$ in place of $\left\langle r^{2}\right\rangle_{*}$

Also $W_{t}^{(k)}(\mathbf{r}), W_{t}^{(k)}(0),\left\langle r^{-1}\right\rangle^{(k)}$ and $P_{t}^{(k)}(\xi)$ are similarly defined. It should be noted that in the above modification the explicit as well as implicit (i.e., through $\left.g_{2 k}^{\prime}\right)\left\langle r^{2}\right\rangle_{*}$ must be replaced with $\left\langle r^{2}\right\rangle$.

Upon integration over angle coordinates, Daniels' distribution function ${ }^{6}$ becomes in the reduced variables

$$
\begin{array}{r}
W_{t *}^{(\mathrm{D})}(\mathbf{r})=\left(\frac{3}{2} \pi t\right)^{-3 / 2} \exp \left(-\frac{3}{2} r^{2} / t\right) \\
\times\left(1-\frac{5}{8 t}+\frac{2 r^{2}}{t^{2}}-\frac{33 r^{4}}{40 t^{3}}\right)
\end{array}
$$

where the superscript $\mathrm{D}$ to $W$ refers, unlike $k$, to Daniels. Originally Daniels' function also was expressed in a series, but terms higher in $t^{-1}$ than those in eq 48 were not given. His series expression corresponds to, and is better compared with, the author's $W_{t *}(\mathbf{r})$, because $\left\langle r^{2}\right\rangle_{*}=t$ for the PK chain. Hence

$$
\begin{aligned}
P_{t *}^{(\mathrm{D})}(\xi)= & 4 \pi\left(\frac{2}{3} \pi\right)^{-3 / 2} \xi^{2} \exp \left(-\frac{3}{2} \xi^{2}\right) \\
& \times\left(1-\frac{5}{8 t}+\frac{2 \xi^{2}}{t}-\frac{33 \xi^{4}}{40 t}\right) \\
& W_{t *}^{(\mathrm{D})}(0)=\left(\frac{2}{3} \pi t\right)^{-3 / 2}\left(1-\frac{5}{8 t}\right) \\
& \left\langle r^{-1}\right\rangle_{t *}^{(\mathrm{D})}=\left(\frac{1}{6} \pi t\right)^{-1 / 2}\left(1-\frac{1}{40 t}\right)
\end{aligned}
$$

In eq $49 \xi=t^{-1 / 2} r$. Daniels' distribution is obtained from $W_{t *}^{(2)}(\mathbf{r})$ by discarding $t^{-2}$ and exponential terms.

\section{RESULTS AND DISCUSSION}

\section{Even Moments}

Calculations were made with a single precision of the computer, i.e., of 40 binary bits, and extended to $k=30$ for both cases (i) and (ii). However, the range of $k$ and $t$ for which the calculated even moments are actually useful is rather limited on account of limited accuracy. In the exponential series expression there occurs a serious cancellation for small $t$, as is apparent from eq 27 and 28 . In the power series expression the series is of slow convergence as well as subject to a cancellation because it is an alternating series, these being more serious for larger values of $k$. Obviously the two expressions supplement each other. There is a range of $t$ within which the accuracy of calculated moments is significantly diminished, or even completely lost, by either method. Such a range increases with increasing $k$. In the present calculations the author was able to cover the entire range of $t$ with an accuracy of $10^{-5}$ or better, but only for values up to $k=6$, i.e., $\left\langle r^{12}\right\rangle$.

$c_{k l j}$ for $\left\langle r^{8}\right\rangle,\left\langle r^{10}\right\rangle$, and $\left\langle r^{12}\right\rangle$ are given in Table I. To obtain these, three runs of computations were carried out which are different in initial values of $c_{111}$ of $u_{1}{ }^{(1)}$ by one or two at the leastsignificant bit. Only those digits which are not affected by these changes are displayed. One sees that the accuracy is considerably diminished for 
some $c_{k l j}$. Fortunately, less accurate $c_{k l j}$ make less contributions to the total $\left\langle r^{2 k}\right\rangle$. (Exact $c_{k l j}$ could be obtained by expressing $c_{k m l j}^{\prime \prime}=\sum_{\mu, \nu} d_{k m l j \mu_{\nu}}$ $\times \mu^{-\nu}$ and storing $d_{k m l j \mu_{\nu}}$ instead of $c_{k m l j}^{\prime \prime}$, where $\mu$ is a positive integer, $\nu$ is zero or a positive integer, and $d_{k m l j \mu_{\nu}}$ is zero or an integer. This procedure requires much more memory, however.)
Comparison with results obtained by the power series method indicates that $c_{k l j}$ in Table I give values of $\left\langle r^{2 k}\right\rangle$ of the accuracy of $10^{-5}$ or better down to $t=0.4,0.6$, and 0.8 for $k=4,5$, and 6 , respectively. For larger $k, t$ must be larger to achieve the same accuracy, e.g., $t>2,12$, and 30 for $k=10,20$, and 30 , respectively, according

Table IIa. $f_{4.0 . n}$ in eq 36

\begin{tabular}{|c|c|c|c|c|c|c|c|c|c|c|}
\hline$n$ & $n$ & & $n$ & & $n$ & & $n$ & & $n$ & \\
\hline 11.199999999 & 10 & 8353347394 & 19 & 29161995 & 280 & 055017 & 37 & 25140 & 46 & 0.8227024752 \\
\hline 21.139999999 & 11 & 308493751 & 20 & 8364856 & 29 & 43491 & 38 & 4989 & 47 & 50 \\
\hline 31.049714285 & 12 & 279325296 & 21 & 7863469 & 30 & 36343 & 39 & 4896 & 48 & 49 \\
\hline 40.9776485714 & 13 & 260461579 & 22 & 7548775 & 31 & 31914 & 40 & 4838 & 49,50 & 48 \\
\hline 50.9266399999 & 14 & 248322522 & 23 & 7351640 & 32 & 29173 & 41 & 4803 & $>50$ & 47 \\
\hline 60.8917836571 & 15 & 240545437 & 24 & 7228369 & 33 & 27478 & 42 & 4781 & & \\
\hline 70.8682937371 & 16 & 235582590 & 25 & 7151412 & 34 & 26431 & 43 & 4768 & & \\
\hline 80.8525991951 & 17 & 232426782 & 26 & 7103441 & 35 & 25785 & 44 & 4760 & & \\
\hline 90.8421901850 & 18 & 230426391 & 27 & 7073580 & 36 & 25386 & 45 & 4755 & & \\
\hline
\end{tabular}

Table IIb. $f_{5.0 . n}$ in eq 36

\begin{tabular}{|c|c|c|c|c|c|c|c|c|c|c|}
\hline$n$ & $n$ & & $n$ & & $n$ & & $n$ & & $n$ & \\
\hline 11.222222222 & 12 & 7395341234 & 23 & 33958505 & 34 & 0834973 & 45 & 84235 & 56 & .7230783480 \\
\hline 21.157037037 & 13 & 347058250 & 24 & 2977716 & 35 & 818701 & 46 & 3990 & 57 & 76 \\
\hline 31.051188712 & 14 & 312709411 & 25 & 2298008 & 36 & 807555 & 47 & 3823 & 58 & 74 \\
\hline 40.9622781892 & 15 & 288354125 & 26 & 1827636 & 37 & 799925 & 48 & 3710 & 59 & 72 \\
\hline 50.8957866666 & 16 & 271139113 & 27 & 1502563 & 38 & 794705 & 49 & 3633 & 60 & 71 \\
\hline 60.8474092117 & 17 & 259006895 & 28 & 1278188 & 39 & 791137 & 50 & 3580 & $61-63$ & 70 \\
\hline 70.8124156432 & 18 & 250480150 & 29 & 1123497 & 40 & 788699 & 51 & 3544 & $64-102$ & 69 \\
\hline 80.7871332471 & 19 & 244502541 & 30 & 1016964 & 41 & 787034 & 52 & 3520 & $>102$ & 6.8 \\
\hline 90.7688864093 & 20 & 240321740 & 31 & 0943673 & 42 & 785898 & 53 & 3504 & & \\
\hline 100.7557419746 & 21 & 237403912 & 32 & 0893298 & 43 & 785124 & 54 & 3492 & & \\
\hline 110.7462980897 & 22 & 235371544 & 33 & 0858706 & 44 & 784595 & 55 & 3485 & & \\
\hline
\end{tabular}

Table IIc. $f_{6.0 . n}$ in eq 36

\begin{tabular}{|c|c|c|c|c|c|c|c|c|c|c|}
\hline \multirow{2}{*}{$\frac{n}{11.238095238}$} & \multicolumn{2}{|l|}{$n$} & \multicolumn{2}{|l|}{$n$} & \multicolumn{2}{|l|}{$n$} & \multicolumn{2}{|l|}{$n$} & \multicolumn{2}{|l|}{$n$} \\
\hline & 140 & 6491781827 & 270 & 6303915245 & 40 & 969588 & 53 & 99790 & 660. & 8658 \\
\hline 21.169312169 & 15 & 443760349 & 28 & 2856324 & 41 & 50435 & 54 & 9476 & 67 & 53 \\
\hline 31.051866249 & 16 & 407559630 & 29 & 2074624 & 42 & 36435 & 55 & 9247 & 68 & 49 \\
\hline 40.9501288043 & 17 & 380324928 & 30 & 1498171 & 43 & 26207 & 56 & 9081 & 69 & 47 \\
\hline 50.8716011853 & 18 & 359876979 & 31 & 1073491 & 44 & 18738 & 57 & 8960 & 70 & 45 \\
\hline 60.8124275277 & 19 & 344554937 & 32 & 0760913 & 45 & 13287 & 58 & 8872 & 71 & 43 \\
\hline 70.7679151708 & 20 & 333095745 & 33 & 0531045 & 46 & 09310 & 59 & 8808 & 72,73 & 42 \\
\hline 80.7343308276 & 21 & 324541141 & 34 & 0362141 & 47 & 06410 & 60 & 8762 & 74,75 & 41 \\
\hline 90.7089157585 & 22 & 318165904 & 35 & 0238129 & 48 & 04296 & 61 & 8728 & $76-112$ & 40 \\
\hline 100.6896467790 & 23 & 313422544 & 36 & 0147144 & 49 & 02756 & 62 & 8704 & $>112$ & 39 \\
\hline 110.6750274062 & 24 & 309898738 & 37 & 0080437 & 50 & 01634 & 63 & 8686 & & \\
\hline 120.6639386341 & 25 & 307284682 & 38 & 0031563 & 51 & 00817 & 64 & 8674 & & \\
\hline 130.6555360227 & 26 & 305348110 & 390 & 6299995776 & 52 & 00223 & 65 & 8664 & & \\
\hline
\end{tabular}


to a comparison of differences in results of the three runs noted above. With the present precision of calculations $\left\langle r^{2 k}\right\rangle$ for $k>20$ are actually useless; they lack accuracies for small $t$ where they are necessary, while they are unnecessary (because of the achieved convergence) for large $t$ where they are sufficiently accurate. For very large $k$, terms for $l=0$ and 1 become dominant over others. For example, for $k=20, c_{20, l j}$ for $l \neq 0$ or 1 are smaller than those for $l=0$ or 1 by a factor of $10^{-13}$ or less. $f_{k 0 n}$ for $k=4,5$, and 6 are given in Tables IIa, b, and c. These $f_{k 0 n}$ give $\left\langle r^{2 k}\right\rangle$ of the accuracy of $10^{-5}$ or better up to $t=1.5,1.2$, and 1.0 for $k=4,5$, and 6 , respectively.

$\left\langle r^{2 k}\right\rangle \mid\left\langle r^{2 k}\right\rangle_{*}$ calculated by the exponential series method are plotted against $t$ with solid curves in Figures 1a and b. Here $\left\langle r^{2 k}\right\rangle_{*}$ is the asymptotic value of $\left\langle r^{2 k}\right\rangle$ at $t=\infty$, i.e.,

$$
\left\langle r^{2 k}\right\rangle_{*}=\frac{(2 k+1) !}{k ! 6^{k}}\left\langle r^{2}\right\rangle_{*}^{k}=\frac{(2 k+1) ! t^{k}}{k ! 6^{k}}
$$

In Figure 1a are included for comparison the previously ${ }^{5}$ calculated results (broken curves) for the realistic, trans-guache rotational-isomericstate model for polyethylene (subchain of ref 5). $n$ in the upper abscissa is the number of $\mathrm{C}-\mathrm{C}$ bonds. It was assumed between $n$ and $t^{*}$

$$
n=12.002 t
$$

* The adjustment of the realistic chain with the PK chain is made as follows. $\left\langle r^{2}\right\rangle$ for very long (say, $n>100$ ) realistic chains ${ }^{5,7}$ and the very long PK chain behave almost exactly as

$$
\begin{array}{ll}
\left\langle r^{2}\right\rangle=A n-B & \text { (realistic chains) } \\
\left\langle r^{2}\right\rangle=2 a L-2 a^{2} & \text { (PK chain) }
\end{array}
$$

The first relation of the adjustment is, as usually made, to equate the leading terms in eq 54 and 55 , i.e., $A n=2 a L$. The second relation proposed here is to equate the second terms, i.e., $B=2 a^{2}$. Then one finds

$$
\begin{aligned}
& a=(B / 2)^{1 / 2} \quad \text { and } \quad L=A(2 B)^{-1 / 2} n \\
& t=L /(2 a)=A(2 B)^{-1} n
\end{aligned}
$$

Numerical results for $A$ and $B$ (taken from eq 66a of reî 5) yielded eq 53. It is found that the adjustment proposed above analytically reproduces an empirical "best shift factor" of Maeda, et al. ${ }^{2}$ Detailed discussions on the adjustment will soon be given elsewhere. ${ }^{11}$

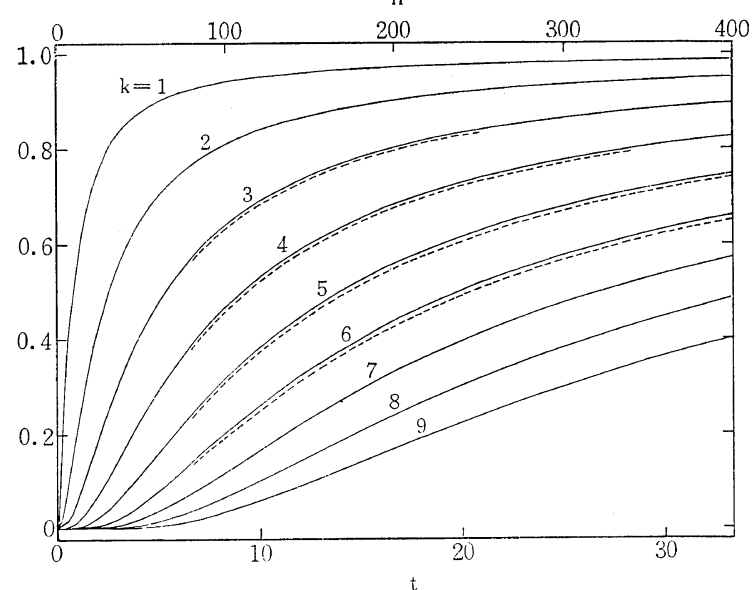

(a)

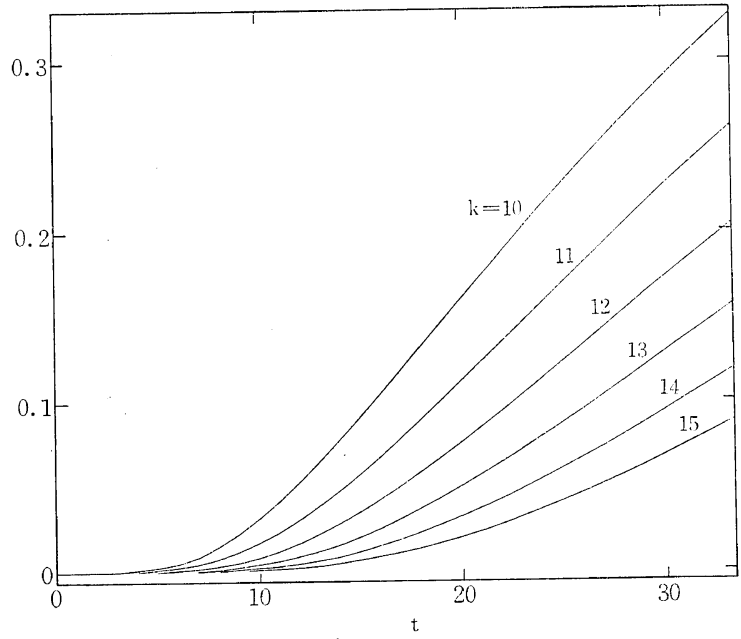

(b)

Figure 1. $\left\langle r^{2 k}\right\rangle\left\langle\left\langle r^{2 k}\right\rangle_{*} v s . t\right.$ for the Porod-Kratky chain (solid curves), and $\left\langle r^{2 k}\right\rangle\left\langle\left\langle r^{2 k}\right\rangle * v s . n\right.$ for the polyethylene chain ${ }^{5}$ (broken curves). $t$ is the contour length, $n$ is the number of $\mathrm{C}-\mathrm{C}$ bonds, and $\left\langle r^{2 k}\right\rangle_{*}$ is the asymptotic value of $\left\langle r^{2 k}\right\rangle$ at $t$ or $n=\infty$. For the adjustment of parameters see text.

Broken curves for $k=1$ and 2 are very close to the corresponding solid curves, and hence are not included to avoid confusion. Though small systematic deviations occur for higher moments, the agreement is very satisfactory and encouraging. $\left\langle r^{2 k}\right\rangle / t^{2 k}$ values calculated with a sufficient accuracy by the power series method are plotted against $t$ in Figure 2. Obviously $t^{2 k}$ is $r^{2 k}$ for the stiff rod. The range of $t$ effectively covered is rather limited, in particular for large $k$, as seen with curves $k=6$ and 10 . The loss of accuracy 


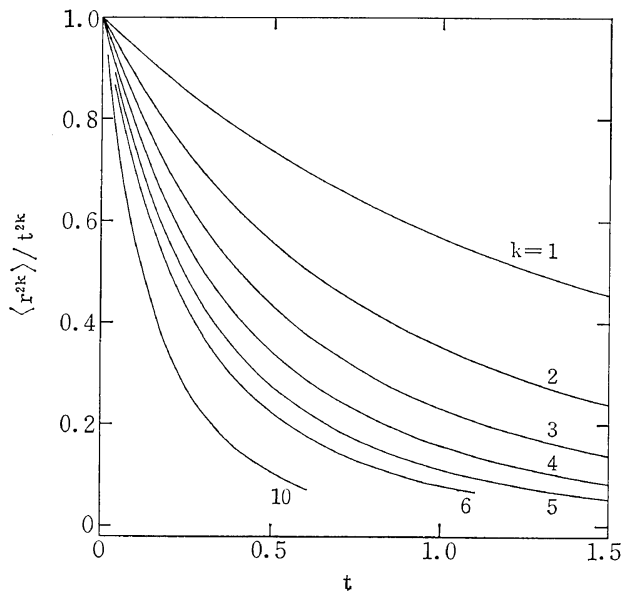

Figure 2. $\left\langle r^{2 k}\right\rangle \mid t^{2 k}$ vs. $t . \quad t^{2 k}$ is $r^{2 k}$ for the stiff rod.

by a cancellation (from summation of terms of alternating sign) was found to be a more serious cause of it than the slow convergence of the series.

$$
W_{t *}(0), W_{t}(0),\left\langle r^{-1}\right\rangle_{*} \text {, and }\left\langle r^{-1}\right\rangle
$$

In Figure 3 and $4, W_{t *}^{(k)}(0) / W_{t *}^{(0)}(0)$ and $W_{t}^{(k)}(0) /$ $W_{t}{ }^{(0)}(0)$ are plotted against $t$. Here the denominators are the respective Gaussian analogs, i.e.,

$$
\begin{aligned}
W_{t *}^{(0)}(0) & =\left(\frac{2}{3} \pi\left\langle r^{2}\right\rangle_{*}\right)^{-3 / 2} \\
\text { and } \quad W_{t}^{(0)}(0) & =\left(\frac{2}{3} \pi\left\langle r^{2}\right\rangle\right)^{-3 / 2}
\end{aligned}
$$

In order to see the speed of convergence, these

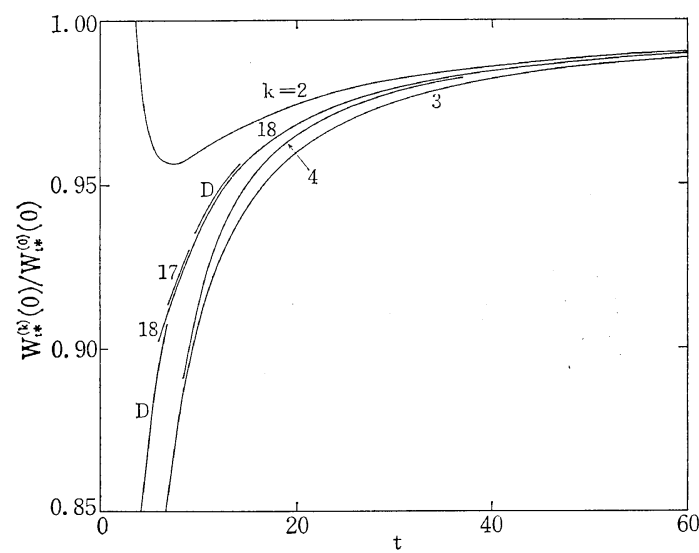

Figgre 3. $W_{t *}^{(k)}(0) / W_{t *}^{(0)}(0) v s$. $t$ for various $k$ indicated. D refers to the consequence of Daniels' distribution, eq 50 .

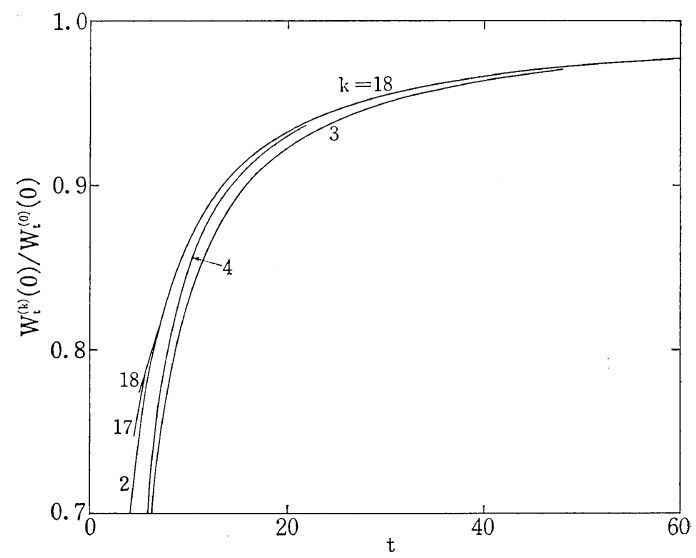

Figure 4. $W_{t}^{(k)}(0) / W_{t}^{(0)}(0) v s$. $t$ for various $k$ indicated.

ratios are plotted against $k$ for selected $t$ in Figure 5. One observes that the covergence, though very protracted, is almost achieved at $k=16-18$ for $t=10$, and at smaller $k$ for larger $t$. Thus one can regard $W_{t *}^{(18)}(0)$ and $W_{t}^{(18)}(0)$ as $W_{t *}(0)$ and $W_{t}(0)$ for $t>10$ in Figures 3 and 4.

It is noteworthy that curve $\mathbf{D}$ for Daniels' distribution is very close to curve 18 in Figure 3 , and curve 2 is very close to curve 18 in Figure 4, suggesting that $W_{t *}^{(\mathrm{D})}(\mathbf{r})$ and $W_{t}^{(2)}(\mathbf{r})$ may be good approximations for $W_{t *}(\mathbf{r})$ and $W_{t}(\mathbf{r})$, respectively. A closer, numerical comparison is made in Table III.

In Figure 6 and $7,\left\langle r^{-1}\right\rangle_{*}^{\left({ }^{(k)}\right.} \mid\left\langle r^{-1}\right\rangle_{*}{ }^{(0)}$ and $\left\langle r^{-1}\right\rangle^{(k)} /\left\langle r^{-1}\right\rangle^{(0)}$ are plotted against $t$, where

and

$$
\begin{aligned}
\left\langle r^{-1}\right\rangle_{*}{ }^{(0)} & =\left(\frac{1}{6} \pi\left\langle r^{2}\right\rangle_{*}\right)^{-1 / 2} \\
\left\langle r^{-1}\right\rangle^{(0)} & =\left(\frac{1}{6} \pi\left\langle r^{2}\right\rangle\right)^{-1 / 2}
\end{aligned}
$$

The general features are almost the same as in the case of $W_{t *}(0)$ and $W_{t}(0)$. The difference between curves $\mathrm{D}$ and 18 in Figure 6 is larger on graphs than that in Figure 4. This is due in most part to the difference in ordinate scales. Numerical results are given in Table IV.

$$
\boldsymbol{P}_{t *}(\xi) \text { and } \boldsymbol{P}_{t}(\xi)
$$

$\boldsymbol{P}_{t *}^{(18)}(\xi)$ and $\boldsymbol{P}_{t}^{(18)}(\xi)$ are plotted against $\xi$ for $t=10,20$, and $\infty$ in Figure 8 and 9 . Different types of plot for $P_{t *}^{(18)}(\xi)$ are shown in Figure 10, i.e., $\log _{10}\left[P_{t *}^{(18)}(\xi) /\left(4 \pi \xi^{2}\right)\right],\left\{=\log _{10}\left[t^{3 / 2} W_{t *}^{(18)}(\mathbf{r})\right]\right\}$, vs. 


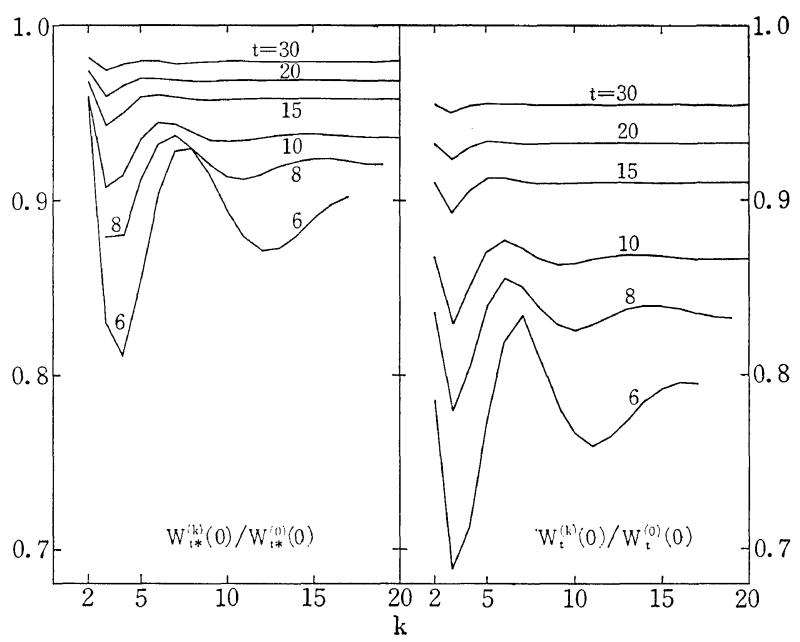

Figure 5. Speeds of convergence of $W_{t *}^{(k)}(0)$ and $W_{t}^{(k)}(0)$ with increasing $k$.

Table III. $W_{t *}(0)$ and $W_{t}(0)$ in various approximations

\begin{tabular}{|c|c|c|c|c|c|c|}
\hline \multirow{2}{*}{$t$} & $W_{t *}^{(18)}(0)$ & $W_{t *}^{(\mathrm{D})}(0)$ & $W_{t *}^{(\mathrm{N})}(0)$ & \multirow{2}{*}{$\frac{W_{t}^{(18)}(0)}{W_{t}^{(0)}(0)}$} & \multirow{2}{*}{$\frac{W_{t}^{(2)}(0)}{W_{t}^{(0)}(0)}$} & \multirow{2}{*}{$\frac{W_{t}^{(\mathrm{N})}(0)}{W_{t}^{(0)}(0)}$, eq 63} \\
\hline & $W_{t *}^{(0)}(0)$ & $W_{t *}^{(0)}(0)$ & $\overline{W_{t *}^{(0)}(0)}$, eq 01 & & & \\
\hline 10 & 0.9355 & 0.93750 & 0.93626 & 0.8660 & 0.86715 & 0.86689 \\
\hline 11 & 416 & 4318 & 4216 & 8781 & 87877 & 87862 \\
\hline 12 & 467 & 4791 & 4705 & 8882 & 88854 & 88846 \\
\hline 13 & 510 & 5192 & 5119 & 8967 & 89686 & 89682 \\
\hline 14 & 546 & 5535 & 5472 & 9039 & 90403 & 90402 \\
\hline 15 & 577 & 5833 & 5778 & 9102 & 91027 & 91028 \\
\hline 16 & 604 & 6093 & 6045 & 9157 & 91575 & 91577 \\
\hline 17 & 627 & 6323 & 6280 & 9206 & 92060 & 92063 \\
\hline 18 & 6488 & 6527 & 6489 & 92496 & 92493 & 92496 \\
\hline 19 & 6675 & 6710 & 6676 & 92884 & 92881 & 92884 \\
\hline 20 & 6843 & 6875 & 6844 & 93234 & 93230 & 93234 \\
\hline 21 & 6994 & 7023 & 6995 & 93551 & 93547 & 93551 \\
\hline 22 & 7132 & 7159 & 7133 & 93840 & 93836 & 93840 \\
\hline 23 & 7258 & 7282 & 7259 & 94104 & 94100 & 94104 \\
\hline 24 & 7373 & 7395 & 7374 & 94346 & 94343 & 94347 \\
\hline 25 & 7479 & 7500 & 7480 & 94569 & 94566 & 94570 \\
\hline 26 & 7577 & 7596 & 7577 & 94776 & 94772 & 94776 \\
\hline 27 & 7667 & 7685 & 7668 & 94967 & 94964 & 94967 \\
\hline 28 & 7751 & 7767 & 7752 & 95144 & 95141 & 95145 \\
\hline 29 & 7829 & 7844 & 7830 & 95310 & 95307 & 95310 \\
\hline 30 & 7902 & 7916 & 7902 & 95464 & 95462 & 95465 \\
\hline
\end{tabular}

$\xi^{2}$, which exaggerate distribution tails. Curve $\mathrm{D}$ is for Daniels' distribution. To examine the degree of convergence $P_{10 *}^{(k)}(\xi)$ and $P_{10}^{(k)}(\xi)$ are plotted against $k$ for selected $\xi$ in Figure 11 . one sees that both functions are almost convergent at $k=16-18$. Accuracies are sufficient for $\xi<2.4$, but are diminished and eventually lost with increasing $\xi$. The functions are more rapidly convergent and accurate up to larger $\xi$ for larger $t$. Therefore, $P_{t *}^{(18)}(\xi)$ and $P_{t}^{(18)}(\xi)$ in the ordinates of Figures 8 and 9 can be regarded as $P_{t *}(\xi)$ and $P_{t}(\xi)$. Similarly, curves $t=10$ and 


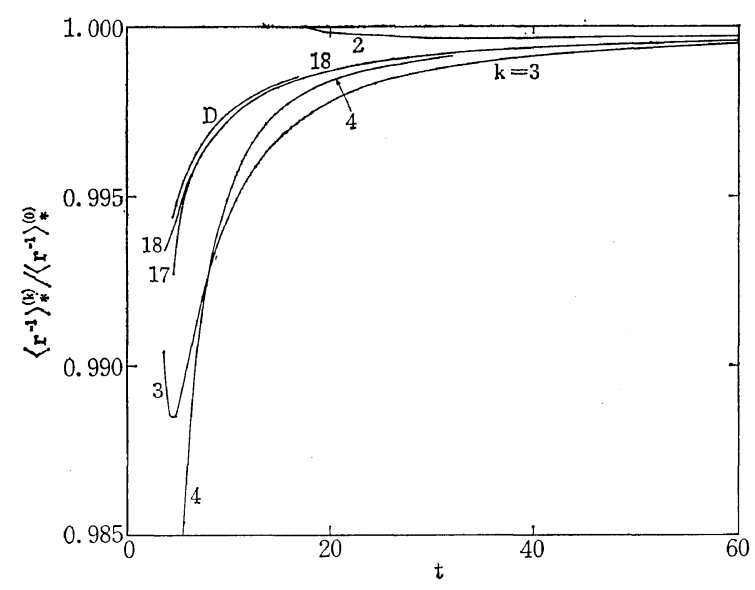

Figure 6. $\left\langle\boldsymbol{r}^{-1}\right\rangle_{*}{ }^{(k)} /\left\langle r^{-1}\right\rangle_{*}{ }^{(0)} v s$. $t$ for various $k$ indicated. D refers to the consequence of Daniels' distribution, eq 51 .

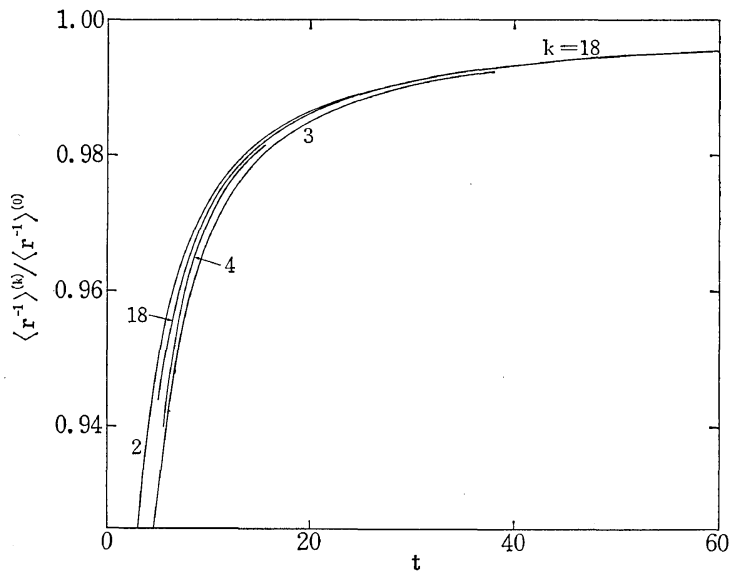

Figure 7. $\left\langle r^{-1}\right\rangle(k) /\left\langle r^{-1}\right\rangle^{(0)}$ vs. $t$ for various $k$ indicated.

Table IV. $\left\langle r^{-1}\right\rangle_{*}$ and $\left\langle r^{-1}\right\rangle$ in various approximations

\begin{tabular}{rrrrrrr}
\hline$t$ & $\frac{\left\langle r^{-1}\right\rangle_{*}^{(18)}}{\left\langle r^{-1}\right\rangle_{*}^{(0)}}$ & $\frac{\left\langle r^{-1}\right\rangle_{*}{ }^{(\mathrm{D})}}{\left\langle r^{-1}\right\rangle_{*}{ }^{(0)}}$ & $\frac{\left\langle r^{-1}\right\rangle_{*}^{(\mathrm{N})}}{\left\langle r^{-1}\right\rangle_{*}{ }^{(0)}}$, eq 62 & $\frac{\left\langle r^{-1}\right\rangle^{(18)}}{\left\langle r^{-1}\right\rangle^{(0)}}$ & $\frac{\left\langle r^{-1}\right\rangle^{(2)}}{\left\langle r^{-1}\right\rangle(0)}$ & $\frac{\left\langle r^{-1}\right\rangle(\mathrm{N})}{\left\langle r^{-1}\right\rangle^{(0)}}$, eq 64 \\
\hline 10 & 0.99730 & 0.997500 & 0.997337 & 0.97204 & 0.973430 & 0.972087 \\
11 & 756 & 7727 & 7592 & 7463 & 75755 & 74658 \\
12 & 778 & 7916 & 7803 & 7677 & 77709 & 76796 \\
13 & 796 & 8076 & 7980 & 7858 & 79373 & 78601 \\
14 & 812 & 8214 & 8131 & 8013 & 80807 & 80146 \\
15 & 825 & 8333 & 8254 & 8147 & 82055 & 81483 \\
16 & 836 & 8437 & 8373 & 8264 & 83151 & 82651 \\
17 & 846 & 8529 & 8473 & 83676 & 84121 & 83680 \\
18 & 8557 & 8611 & 8560 & 84591 & 84986 & 84594 \\
19 & 8636 & 8684 & 8639 & 85408 & 85762 & 85411 \\
20 & 8706 & 8750 & 8709 & 86144 & 86461 & 86146 \\
21 & 8770 & 8809 & 8772 & 86808 & 87095 & 86811 \\
22 & 8828 & 8863 & 8829 & 87412 & 87673 & 87414 \\
23 & 8880 & 8913 & 8882 & 87963 & 88201 & 87965 \\
24 & 8928 & 8958 & 8930 & 88468 & 88686 & 88469 \\
25 & 8972 & 9000 & 8973 & 88932 & 89133 & 88933 \\
26 & 9013 & 9038 & 9014 & 89360 & 89545 & 89361 \\
27 & 9050 & 9074 & 9051 & 89757 & 89928 & 89758 \\
28 & 9085 & 9107 & 9086 & 90124 & 90283 & 90125 \\
29 & 9117 & 9137 & 9118 & 90467 & 90615 & 90468 \\
30 & 9147 & 9166 & 9148 & 90786 & 90924 & 90787 \\
\hline
\end{tabular}

20 in Figure 10 can be regarded as for $\boldsymbol{P}_{t *}(\xi)$. smaller than those of $\boldsymbol{P}_{t *}^{(18)}(\xi)$ from $\boldsymbol{P}_{t *}^{(0)}(\xi)$. This Open circles in Figure 11 are for Daniels' dis- can be seen clearly with the following numerical tribution. They are close to the asymptotic, values: $\xi, P_{t *}^{(0)}(\xi), P_{10 *}^{(18)}(\xi), P_{10 *}^{(\mathrm{D})} ; 0.4,0.52180$, exact values for $\xi<1.0$, but discernibly deviate $0.50345,0.50479 ; 0.8,1.01596,1.04964,1.04818$, for larger $\xi$ (see also Figure 10). However, 1.2, 0.68850, 0.73334, 0.72598; 1.6, 0.22812, deviations of $P_{t *}^{(18)}(\xi)$ from $P_{t *}^{(D)}(\xi)$ are much $0.19972,0.20732 ; 2.4,0.0042241,0.000022$, 


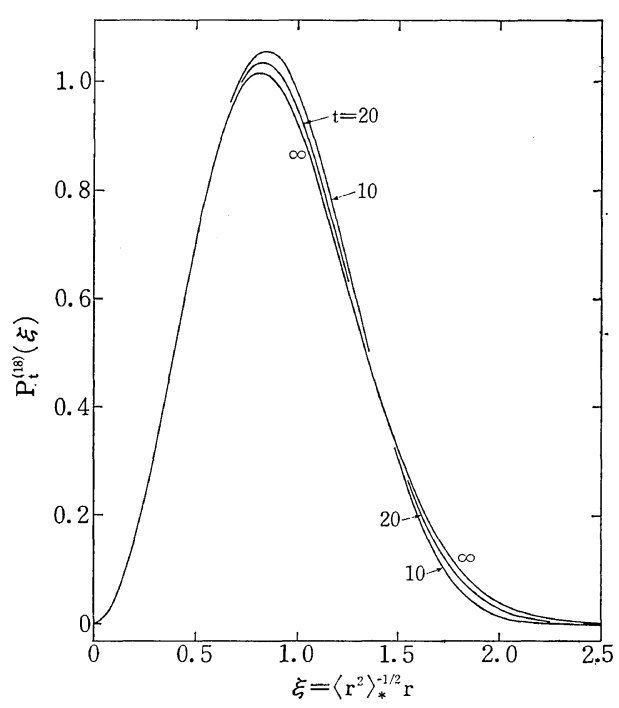

Figure 8. $P_{t *}^{(18)}(\xi) v s$. $\xi$ for $t$ indicated.

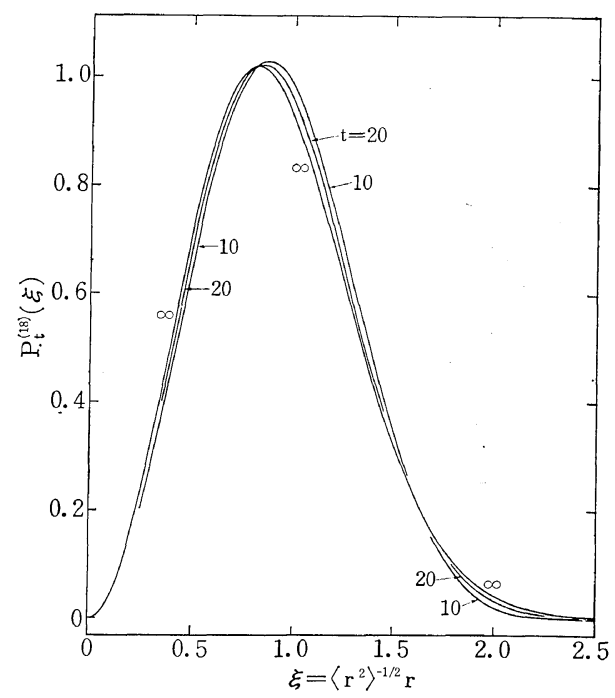

Figgre 9. $P_{t}^{(18)}(\xi)$ vs. $\xi$ for $t$ indicated.

-0.0027357. Thus $P_{t *}^{(D)}(\xi)$ is a good approximation for $P_{t *}(\xi)$ for $\xi<2.4$ and $t=10$, and better up to larger $\xi$ for larger $t$. The situation is similar for $\boldsymbol{P}_{t}^{(2)}(\xi)$ as an approximation for $\boldsymbol{P}_{t}(\xi)$. Degrees of approximation of $\boldsymbol{P}_{t *}^{(\mathrm{D})}(\xi)$ and $\boldsymbol{P}_{t}{ }^{(2)}(\xi)$ are not much different if the whole range of $\xi<2.4$ is considered. $\boldsymbol{P}_{t *}^{(\mathrm{D})}(\xi)$ is preferred on account of its simplicity.

The author attempted to improve Daniels' distribution by including higher-order terms. In

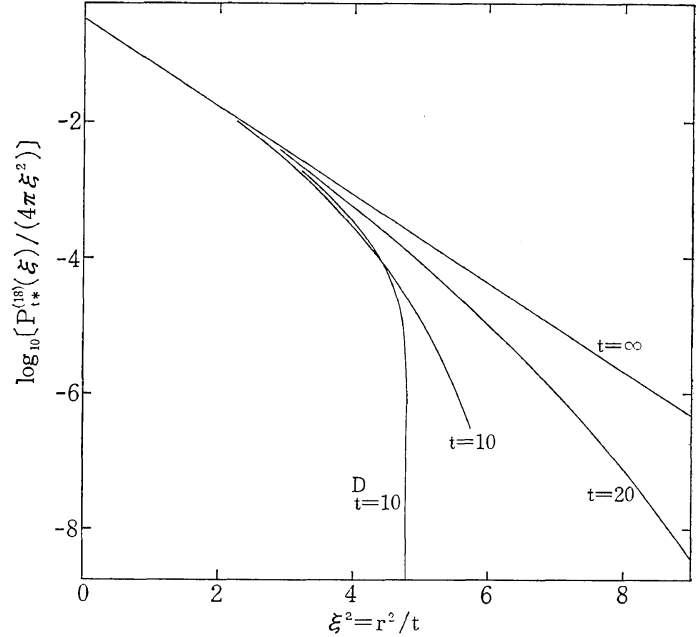

Figure 10. $\log _{10}\left[P_{* t}^{(18)}(\xi) /\left(4 \pi \xi^{2}\right)\right] v s$. $\xi^{2}$ for $t$ indicated. Curve D, $t=10$ is for Daniels' distribution. Curve $\mathrm{N}, t=10$ for eq 60 , not given, falls between curve $t=10$ and curve $\mathrm{D}, t=10$, being closer to the former for $\xi^{2}<4.5$ and the latter for $\xi^{2}>4.5$.

this connection the following point should be noted $W_{t *}^{(2)}(\mathbf{r})$ yields exact, second and fourth moments while $W_{t *}^{(\mathrm{D})}(\mathbf{r})$ gives approximate ones. $\left[W_{t *}^{(\mathrm{D})}(\mathbf{r})\right.$ gives even moments made up of two terms, these being exactly the same as the leading two terms in exact even moments: for example, $\left\langle r^{4}\right\rangle=(5 / 3) t^{2}-(26 / 9) t$ instead of eq $\left.28 .{ }^{12}\right]$ Nevertheless, $W_{t *}^{(\mathbf{D})}(\mathbf{r})$ is better than $W_{t *}^{(2)}(\mathbf{r})$. Exponential terms in even moments are negligible for $t>5$. The difference between $W_{t *}^{(\mathrm{D})}(\mathbf{r})$ and $W_{t *}^{(2)}(\mathbf{r})$ is that the former contains all of the $t^{-1}$ terms and $n o$ higher terms in $W_{t *}(\mathbf{r})$, whereas the latter has all of the $t^{-1}$ terms and part of the $t^{-2}$ terms in $W_{t *}(\mathbf{r})$. This fact suggests that an improvement could be made by retaining all of $t^{-1}$ and $t^{-2}$ terms and by discarding all higher-order terms in $W_{t *}(\mathbf{r})$. To this end one must take into account up to the $k=4$ term in eq $37 .^{7,12}$ Hence

$$
\begin{aligned}
W_{t *}^{(\mathrm{N})}(\mathbf{r})= & \left(\frac{3}{2} \pi t\right)^{-3 / 2} \exp \left(-\frac{3}{2} r^{2} / t\right) \\
& \times\left[1-\frac{1}{8 t}\left(5+\frac{7.9}{8 t}\right)+\left(2-\frac{32.9}{24 t}\right) \frac{r^{2}}{t^{2}}\right. \\
& +\left(-\frac{3.3}{4}+\frac{67.99}{16 t}\right) \frac{r^{4}}{t^{3}}-\frac{34.41}{14} \frac{t^{6}}{t^{5}} \\
& \left.+\frac{10.89}{32} \frac{r^{8}}{t^{6}}\right]
\end{aligned}
$$




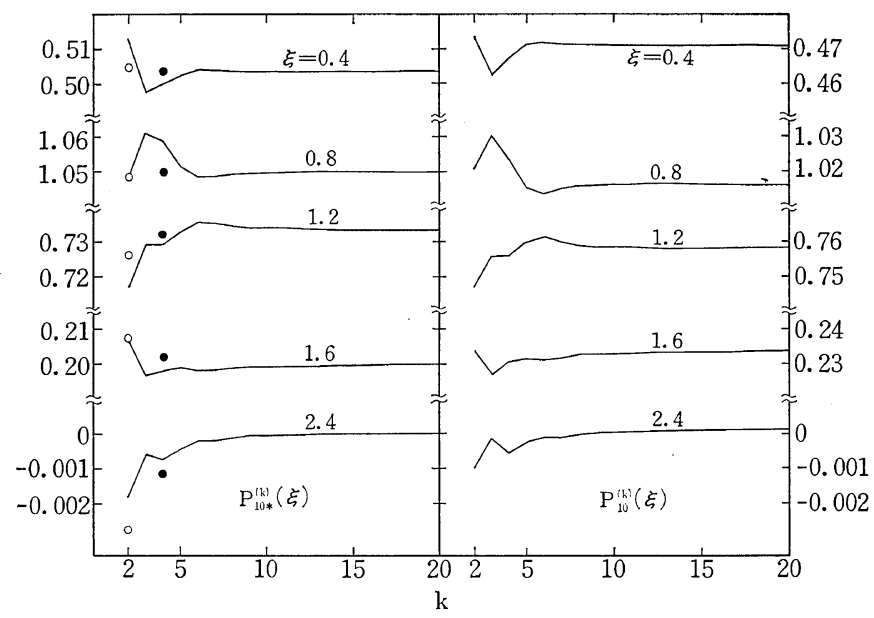

Figure 11. Convergence of $\boldsymbol{P}_{10 *}^{(k)}(\xi)$ and $\boldsymbol{P}_{10}^{(k)}(\xi)$ with increasing $k$ for selected $\xi$. Open circles denote predictions of Daniels' distribution, eq 49 , and filled ones those of eq 60 [of course converted to $P_{t *}^{(\mathrm{N})}(\xi)$ ].

$$
\begin{aligned}
& W_{t *}^{(\mathrm{N})}(0)=\left(\frac{3}{2} \pi t\right)^{-3 / 2}\left[1-\frac{1}{8 t}\left(5+\frac{7.9}{8 t}\right)\right] \\
& \left\langle r^{-1}\right\rangle_{*}^{(\mathrm{N})}=\left(\frac{1}{6} \pi t\right)^{-1 / 2}\left(1-\frac{1}{40 t}-\frac{7.3}{448 t^{2}}\right)
\end{aligned}
$$

where the superscript $\mathrm{N}$ refers to Nagai, not do numerals. Numerical coefficients in eq $60-62$ are exact. $W_{t *}^{(\mathrm{N})}(\mathbf{r})$ gives even moments composed of three terms, these being exactly the same as the leading three terms in exact even moments. $P_{t *}^{(N)}(\xi)$ is defined from $W_{t *}^{(\mathbb{N})}(\mathbf{r})$ by patterning after $\boldsymbol{P}_{t *}^{(k)}(\xi)$ and $\boldsymbol{P}_{t *}^{(\mathrm{D})}(\xi)$. Values of $\boldsymbol{P}_{10 *}^{(\mathrm{N})}(\xi)$ are shown by filled circles in Figure 11 (see also the caption for Figure 10$). \quad \boldsymbol{P}_{t *}^{(\mathrm{N})}(\xi)$ is definitely an improvement over Daniels' distribution $\boldsymbol{P}_{t *}^{(\mathrm{D})}(\xi) . \quad \boldsymbol{P}_{t *}^{(\mathrm{N})}(\xi)$ is better than $\boldsymbol{P}_{t *}^{(4)}(\xi)$ despite the fact that the former gives approximate even moments whereas the latter gives exact even moments up to $\left\langle r^{8}\right\rangle$. Unasterisked counterparts of eq 61 and 62 can be obtained by writing $t$ neighboring $\pi$ in these expressions as

$$
\begin{aligned}
t & =\left(t-\frac{1}{2}\right)\left[1+\frac{1}{2 t}\left(1-\frac{1}{2 t}\right)^{-1}\right] \\
& =\left(t-\frac{1}{2}\right)\left(1+\frac{1}{2 t}+\frac{1}{4 t^{2}}+\cdots\right)
\end{aligned}
$$

i.e.,

$W_{t}^{(\mathrm{N})}(0)=\left[\frac{3}{2} \pi\left(t-\frac{1}{2}\right)\right]^{-3 / 2}\left[1-\frac{1}{8 t}\left(11-\frac{28.1}{8 t}\right)\right]$

$$
\left\langle r^{-1}\right\rangle^{(N)}=\left[\frac{1}{6} \pi\left(t-\frac{1}{2}\right)\right]^{-1 / 2}\left(1-\frac{11}{40 t}-\frac{18.5}{448 t^{2}}\right)
$$

where higher than $t^{-2}$ terms are discarded. Numerical results based on eq $61-64$ are included in Tables III and IV.*

It was $\operatorname{argued}^{9,10}$ that eq 44 is paefered over eq 37 on account of the rapid convergence. Present calculations (Figure 11) indicate that speeds of convergence of both series are almost identical for the PK chain.

* It is found that numerical data are a little better fitted by the following equations than by eq $61-64$ :

$$
\begin{aligned}
& W_{t *}^{(\mathrm{N})}(0)=\left(\frac{3}{2} \pi t\right)^{-3 / 2-}\left[1-\frac{1}{8 t}\left(5+\frac{1}{t}\right)\right] \\
& \left\langle r^{-1}\right\rangle_{*}^{(\mathrm{N})}=\left(\frac{1}{6} \pi t\right)^{-1 / 2}\left[1-\frac{1}{120 t}\left(3+\frac{2}{t}\right)\right] \\
& W_{t}^{(\mathrm{N})}(0)=\left[\frac{3}{2} \pi\left(t-\frac{1}{2}\right)\right]^{-3 / 2}\left[1-\frac{1}{16 t}\left(22-\frac{7}{t}\right)\right] \\
& \left\langle r^{-1}\right\rangle^{(\mathrm{N})}=\left[\frac{1}{6} \pi\left(t-\frac{1}{2}\right)\right]^{-1 / 2-}\left[1-\frac{1}{120 t}\left(33+\frac{5}{t}\right)\right]
\end{aligned}
$$

The author cannot be sure whether this result is real, i.e., eq 65-68 are really better, or just a consequence of some systematic errors in the computed results. Analytical eq 61-64 are preferred over empirical eq 65-68. 


\section{CONCLUSIONS}

A general method for calculating even moments of the PK chain has been developed by adapting Hermans and Ullman's method to computer calculations, and these have actually been calculated up to $\left\langle r^{40}\right\rangle$. A good agreement is found between these even moments and those for a realistic model for polyethylene, if a proper adjustment of parameters is made.

These even moments have been used to calculate distribution functions and related quantities. The series are almost convergent at $k=16-18$ for $t=10$ and at lower $k$ for larger $t$. This work is the first in which the series, eq 37 and 44 , are calculated until a sufficient convergence is reached. Equations 37 and 44 are not tractable, but are unique, exact expressions available for models of physical interest, ${ }^{7,10,12}$ except for the simplest, i.e., the random flight chain. ${ }^{13}$ If even moments are calculable for a model chain, they serve as a basis for judging the validity and usefulness of any approximate distribution functions, as done by us with Daniels' distribution for the PK chain.

Daniels' distribution is found to be satisfactory approximation despite its simplicity. $W_{t}^{(2)}(\mathbf{r})$ is equally good. $W_{t *}^{(\mathrm{N})}(\mathbf{r})$, derived in this paper, is better than both.

\section{REFERENCES}

1. G. Porod, Monatsh Chem., 80, 251 (1949); O. Kratky and G. Porod, Rec. Trav. Chim., 68, 1106 (1949).

2. H. Maeda, N. Saito, and W. H. Stockmayer, Polymer J., 2, 94 (1971).

3. J. J. Hermans and R. Ullman, Physica, 18, 951 (1952).

4. S. Heine, O. Kratky, G. Porad, and P. J. Schmitz, Makromol. Chem., 44, 682 (1961).

5. K. Nagai, J. Chem. Phys., 48, 5646 (1968).

6. H. E. Daniels, Proc. Roy. Soc. Edinburgh, 63, 290 (1952).

7. K. Nagai, J. Chem. Phys., 38, 924 (1963).

8. K. Nagai, J. Chem. Phys., 45, 838 (1966).

9. R. L. Jernigan and P. J. Flory, J. Chem. Phys., 50, 4185 (1969).

10. P. J. Flory, "Statistical Mechanics of Chain Molecules," Interscience, New York, N. Y., 1969, Chapter VIII.

11. K. Nagai, in preparation.

12. T. M. Birshtein and O. B. Ptitsyn, "Conformations of Macromolecules," translated by S. N. Timasheff and M. J. Timasheff, Interscience, New York, N. Y., 1966, Chapter 5.

13. L. R. G. Treloar, Trans. Faraday Soc., 42, 77 (1946); "The Physics of Rubber Elasticity," 2nd ed., Oxford Univ. Press, 1958, p 108. 